\title{
On Ideal Convergence of Sequences of Functions in Intuitionistic Fuzzy Normed Spaces
}

\author{
Vatan KARAKAYA ${ }^{1, *}$, Necip ŞIMMŞEK ${ }^{2}$, Müzeyyen ERTÜRK $K^{3}$ and Faik GÜRSOY3 \\ ${ }^{1}$ Department of Mathematical Engineering, Yildiz Technical University, Davutpasa Campus, Esenler-İstanbul, Turkey \\ ${ }^{2}$ Department of Mathematics, İstanbul Commerce University, Üsküdar-İstanbul, Turkey \\ ${ }^{3}$ Department of Mathematics, Yildiz Technical University, Davutpasa Campus, Esenler-İstanbul, Turkey
}

Received: 28 Aug. 2013, Revised: 25 Nov. 2013, Accepted: 26 Nov. 2013

Published online: 1 Sep. 2014

\begin{abstract}
In this work, our purpose is to introduce $I$-convergence of sequences of functions in intuitionistic fuzzy normed space by combining the $I$-convergence, the sequences of functions and the intuitionistic fuzzy normed spaces, and to investigate relations among concepts such as $I$-convergence, statistical convergence and the usual convergence of sequences of functions in intuitionistic fuzzy normed space.
\end{abstract}

Keywords: I-Uniform convergence, Sequences of function, Intuitionistic Fuzzy Normed Space.

\section{Introduction and Preliminaries}

The fuzzy theory has emerged as the most active area of research in many branches of mathematics and engineering. This new theory was introduced by Zadeh [29] in 1965 and since then a large number of research papers have appeared by using the concept of fuzzy set/numbers and fuzzification of many classical theories has also been made. It has also very useful applications in various fields, e.g. population dynamics [3], chaos control [6], computer programming [8], nonlinear dynamical systems [9], fuzzy physics [17], fuzzy topology ([24],[26]), etc. As a generalization of fuzzy sets, the concept of intuitionistic fuzzy sets was introduced by Atanassov [1] in 1986 and later there has been much progress in the study of intuitionistic fuzzy sets (IFS for short) by many authors including Mursaleen et al [19] and Mursaleen et al [18]. Using the idea of intuitionistic fuzzy sets, Park [25] defined the notion of intuitionistic fuzzy metric spaces by the help of the continuous t-norms and the continuous t-conorms as a generalization of fuzzy metric space due to George and Veeramani [7]. Afterward, Samanta and Jebril [27] introduced the definition of intuitionistic fuzzy continuity and sequentially intuitionistic fuzzy continuity and proved that they are equivalent. A few of the algebraic and topological properties of intutionistic fuzzy continuity and uniformly intutionistic fuzzy continuity was investigated by Dinda and Samanta [4].

On the other hand, the notion of statistical convergence for real number sequences was first introduced by Fast [5]. Using the concept of an ideal, Kostyrko et al. [15] introduced the notion of ideal convergence which is a generalization of ordinary convergence and statistical convergence. The ideal convergence provides a general framework to study the properties of various types of convergence. Additionally, Karakuş et al. [13] defined statistical convergence in intuitionistic fuzzy normed spaces (IFNS for short) and Mursaleen et al. [22] investigated statistical convergence of double sequences in IFNS. Quite recently, in [10], Karakaya et al defined and studied statistical convergence of sequences of functions in IFNS. Furthermore, Karakaya et al ([11], [12]) studied $\lambda$-statistical convergence and lacunary statistical convergence of sequences of functions in IFNS respectively. Further information on ideal convergence and statistical convergence can be found in ([20], [21], [14], [23]).

In this work, our purpose is to introduce some new concepts related to ideal convergence of sequences of functions in IFNS. Also we investigate some relations among the other concepts such as the statistical convergence in classical sense, the statistical convergence

\footnotetext{
*Corresponding author e-mail: vkkaya@yahoo.com, vkkaya@yildiz.edu.tr
} 
in IFNS and statistical convergence of sequences of functions in IFNS.

We first recall some basic notions of IFNS.

\section{Definition 1(see [28]).}

A binary operation $*:[0,1] \times[0,1] \rightarrow[0,1]$ is said to be a continuous $t$-norm if it satisfies the following conditions:

$(i) *$ is associative and commutative,

(ii) $*$ is continuous,

(iii) $a * 1=$ a for all $a \in[0,1]$,

(iv) $a * c \leq b * d$ whenever $a \leq b$ and $c \leq d$ for each $a, b, c, d \in[0,1]$.

For example, $a * b=a . b$ is a continuous t-norm.

A binary operation $\diamond:[0,1] \times[0,1] \rightarrow[0,1]$ is said to be a continuous t-conorm if it satisfies the following conditions:

$(i) \diamond$ is associative and commutative,

(ii) $\diamond$ is continuous,

(iii) $a \diamond 0=$ a for all $a \in[0,1]$

(iv) $a \diamond c \leq b \diamond d$ whenever $a \leq b$ and $c \leq d$ for each $a, b, c, d \in[0,1]$.

For example, $a \diamond b=\min \{a+b, 1\}$ is a continuous tnorm.

\section{Definition 2(see [26]).}

Let $*$ be a continuous $t$-norm, $\diamond$ be a continuous $t$-conorm and $X$ be a linear space over the field $I F(\mathbb{R}$ or $\mathbb{C})$. If $\mu$ and $v$ are fuzzy sets on $X \times(0, \infty)$ satisfying the following conditions, the five-tuple $(X, \mu, v, *, \diamond)$ is said to be an IFNS and $(\mu, v)$ is called an intuitionistic fuzzy norm. For every $x, y \in X$ and $s, t>0$,

(i) $\mu(x, t)+v(x, t) \leq 1$,

(ii) $\mu(x, t)>0$,

(iii) $\mu(x, t)=1 \Longleftrightarrow x=0$,

(iv) $\mu(a x, t)=\mu\left(x, \frac{t}{|a|}\right)$ for each $a \neq 0$,

(v) $\mu(x, t) * \mu(y, s) \leq \mu(x+y, t+s)$,

$(v i) \mu(x,):.(0, \infty) \rightarrow[0,1]$ is continuous

(vii) $\lim _{t \rightarrow \infty} \mu(x, t)=1$ and $\lim _{t \rightarrow 0} \mu(x, t)=0$,

(viii) $v(x, t)<1$,

(ix) $v(x, t)=0 \Longleftrightarrow x=0$,

(x) $v(a x, t)=v\left(x, \frac{t}{|a|}\right)$ for each $a \neq 0$,

(xi) $v(x, t) \diamond v(y, s) \geq v(x+y, t+s)$,

(xii) $v(x,):.(0, \infty) \rightarrow[0,1]$ is continuous.

(xiii) $\lim _{t \rightarrow \infty} v(x, t)=1$ and $\lim _{t \rightarrow 0} v(x, t)=0$,
For the intuitionistic fuzzy normed space $(X, \mu, v, *, \diamond)$, as given in Dinda and Samanta [4], we further assume that $(X, \mu, v, *, \diamond)$, satisfy the following axiom: (xiv) $a * a=a, a \diamond a=a$ for all $a \in[0,1]$.

\section{Definition 3(see [26]).}

Let $(X, \mu, v, *, \diamond)$ be IFNS and $\left(x_{n}\right)$ be sequence in $X$. Sequence $\left(x_{n}\right)$ is said to be convergent to $L \in X$ with respect to the intuitionistic fuzzy norm $(\mu, v)$ if for every $\varepsilon>0$ and $t>0$, there exists a positive integer $n_{0}$ such that $\mu\left(x_{n}-L, t\right)>1-\varepsilon$ and $v\left(x_{n}-L, t\right)<\varepsilon$ whenever $n>n_{0}$. In this case we write $(\mu, v)-\lim x_{n}=L$ as $n \rightarrow \infty$.

\section{Definition 4(see [4]).}

Let $f_{n}:(X, \mu, v, *, \diamond) \rightarrow\left(Y, \mu^{\prime}, \nu^{\prime}, *, \diamond\right)$ be a sequence of functions. The sequence $\left(f_{n}\right)$ is said to be pointwise intuitionistic fuzzy convergent on $X$ to a function $f$ with respect to $(\mu, v)$ if for each $x \in X$, the sequence $f_{n}(x)$ is convergent to $f(x)$ with respect to $\left(\mu^{\prime}, v^{\prime}\right)$.

Let $f_{n}:(X, \mu, v, *, \diamond) \rightarrow\left(Y, \mu^{\prime}, v^{\prime}, *, \diamond\right)$ be a sequence of functions. The sequence $\left(f_{n}\right)$ is said to be uniformly intuitionistic fuzzy convergent on $X$ to a function $f$ with respect to $(\mu, v)$, if $0<r<1, t>0$, there exist a positive integer $n_{0}=n_{0}(r, t)$ such that $\forall x \in X$ and $\forall n>n_{0}$,

$$
\mu\left(f_{n}(x)-f(x), t\right)>1-r, v\left(f_{n}(x)-f(x), t\right)<r .
$$

Definition 5. If $X$ be a non-empty set, then a family of set $I \subset P(X)$ is called an ideal in $X$ if and only if

(i) $\emptyset \in I$;

(ii) For each $A, B \in I$, we have $A \cup B \in I$;

(iii) For each $A \in I$ and $B \subset A$, we have $B \in I$.

Definition 6. Let $X$ be a non-empty set. A non-empty family of sets $F \subset P(X)$ is called a filter on $X$ if and only if

(i) $\emptyset \notin F$;

(ii) For each $A, B \in F$ we have $A \cap B \in F$;

(iii) For each $A \in F$ and $A \subset B$ we have $B \in F$.

An ideal $I$ is called non-trivial if $I \neq \emptyset$ and $X \notin I$. Also a non-trivial ideal $I \subset P(X)$ is called an admissible ideal in $X$ if and only if it contains all singletons i.e., if it contains $\{\{x\}: x \in X\}$.

\section{Definition 7(see [16]).}

Let $I \subset P(\mathbb{N})$ be a nontrivial ideal and $(X, \mu, v, *, \diamond)$ be an IFNS. A sequence $x=\left(x_{n}\right)$ of elements in $X$ is said to I-convergent to $L \in X$ with respect to the intuitionistic fuzzy norm $(\mu, v)$ if for each $\varepsilon>0$ and $t>0$, the set

$$
\left\{n \in \mathbb{N}: \mu\left(x_{n}-L, t\right) \leq 1-\varepsilon \text { or } v\left(x_{n}-L, t\right) \geq \varepsilon\right\} \in I .
$$

In this case the element $L$ is called I-limit of the sequence $\left(x_{n}\right)$ with respect to the intuitionistic fuzzy norm $(\mu, v)$ and we write $I_{(\mu, v)}-\operatorname{limx} x_{n}=L$. 


\section{$2 I$-convergence of sequences of functions in an $I F N S$}

In this section, we define $I$-convergence of sequences of functions in an IFNS and we give some connections between $I$-convergence and statistical convergence of sequences of functions in an IFNS.

Definition 8.Let $I \subset P(\mathbb{N})$ be a nontrival ideal. Also let $(X, \mu, v, *, \diamond), \quad\left(Y, \mu^{\prime}, v^{\prime}, *, \diamond\right)$ be two IFNS and $f_{n}:(X, \mu, v, *, \diamond) \rightarrow\left(Y, \mu^{\prime}, v^{\prime}, *, \diamond\right)$ be a sequence of functions. If for each $x \in X$ and $\forall \varepsilon>0, t>0$,

$$
\begin{array}{r}
\left\{n \in \mathbb{N}: \mu^{\prime}\left(f_{n}(x)-f(x), t\right) \leq 1-\varepsilon\right. \\
\left.\quad \text { or } v^{\prime}\left(f_{n}(x)-f(x), t\right) \geq \varepsilon\right\} \in I
\end{array}
$$

then we say that the sequence $\left(f_{n}\right)$ is pointwise $I-$ ideal convergent with respect to intuitionistic fuzzy norm $(\mu, v)$ and we write it $I_{(\mu, v)}-f_{n} \rightarrow f$.

Example 1. Let $A=\{1,3,5, \ldots, 2 k+1, \ldots\}$ and $I=P(A)=$ $2^{A}$. $I$ is a non trivial ideal in $\mathbb{N}$. Let $(\mathbb{R},|\cdot|)$ denote the space of real numbers with the usual norm, and let $a * b=a . b$ and $a \diamond b=\min \{a+b, 1\}$ for $a, b \in[0,1]$. For all $x \in \mathbb{R}$ and every $t>0$, we consider

$$
\mu(x, t)=\frac{t}{t+|x|} \text { and } v(x, t)=\frac{|x|}{t+|x|} .
$$

In this case, $(\mathbb{R}, \mu, v, *, \diamond)$ is intuitionistic fuzzy normed space. Let $f_{n}:[0,1] \rightarrow \mathbb{R}$ be sequences of functions whose terms are given by

$$
f_{n}(x)=\left\{\begin{array}{cc}
x^{n^{2}}+1 ; & \text { if } n=2 k+1 \text { and } x \in\left[0, \frac{1}{2}\right) \\
0 ; & \text { if } n \neq 2 k+1 \text { and } x \in\left[0, \frac{1}{2}\right) \\
x^{n}+\frac{1}{2} ; & \text { if } n=2 k+1 \text { and } x \in\left[\frac{1}{2}, 1\right) \\
1 ; & \text { if } n \neq 2 k+1 \text { and } x \in\left[\frac{1}{2}, 1\right) \\
2 ; & \text { if } x=1
\end{array}\right.
$$

$\left(f_{n}\right)$ is pointwise $I-$ convergent on $[0,1]$ with respect to intuitionistic fuzzy norm $(\mu, v)$. If we take $x \in\left[0, \frac{1}{2}\right)$ and after the following calculations

$$
\begin{aligned}
& K(\varepsilon, t)=\left\{n \in \mathbb{N}: \frac{t}{t+\left|f_{n}(x)-0\right|} \leq 1-\varepsilon\right. \\
&\left.\quad \text { or } \frac{\left|f_{n}(x)-0\right|}{t+\left|f_{n}(x)-0\right|} \geq \varepsilon\right\} \\
&=\left\{n \in \mathbb{N}:\left|f_{n}(x)\right| \geq \frac{\varepsilon t}{1-\varepsilon}\right\} \\
&=\left\{n \in \mathbb{N}: f_{n}(x)=x^{n^{2}}+1\right\} \\
&=\{n \in \mathbb{N}: n=2 k+1\}
\end{aligned}
$$

we have $K(\varepsilon, t) \in I$. Hence $I_{(\mu, v)}-f_{n} \rightarrow 0$ in an IFNS. If we take $x \in\left[\frac{1}{2}, 1\right)$, then we get the following set

$$
\begin{aligned}
& K^{\prime}(\varepsilon, t)=\left\{n \in \mathbb{N}: \frac{t}{t+\left|f_{n}(x)-1\right|} \leq 1-\varepsilon\right. \\
&\left.\quad \text { or } \frac{\left|f_{n}(x)-1\right|}{t+\left|f_{n}(x)-1\right|} \geq \varepsilon\right\} \\
&=\left\{n \in \mathbb{N}:\left|f_{n}(x)-1\right| \geq \frac{\varepsilon t}{1-\varepsilon}\right\} \\
&=\left\{n \in \mathbb{N}: f_{n}(x)=x^{n}+\frac{1}{2}\right\} \\
&=\{n \in \mathbb{N}: n=2 k+1\} .
\end{aligned}
$$

In this interval, the set $K^{\prime}(\varepsilon, t) \in I$ and $I_{(\mu, v)}-f_{n} \rightarrow 1$ in an IFNS. If we take $x=1$, it can be seen easily that

$$
\begin{aligned}
& K^{\prime \prime}(\varepsilon, t)=\left\{n \in \mathbb{N}: \frac{t}{t+\left|f_{n}(x)-2\right|} \leq 1-\varepsilon\right. \\
&\left.\quad \text { or } \frac{\left|f_{n}(x)-2\right|}{t+\left|f_{n}(x)-2\right|} \geq \varepsilon\right\} \\
&=\left\{n \in \mathbb{N}: 0 \geq \frac{\varepsilon t}{1-\varepsilon}\right\} \\
&=\oslash \in I
\end{aligned}
$$

and $\left(f_{n}\right)$ is ideal convergent to 2 at $x=1$ with respect to intuitionistic fuzzy norm $(\mu, v)$. Since sequence $\left(f_{n}\right)$ has three different limits, it is pointwise $I-$ convergent in $(\mathbb{R}, \mu, v, *, \diamond)$ for each $x \in X_{0}$.

Remark. In the example above, $\left(f_{n}\right)$ is not pointwise convergent both usual sense and statistical sense with respect to $(\mu, v)$ but it is pointwise $I$-convergent with respect to $(\mu, v)$.

Lemma 1. Let $f_{n}:(X, \mu, v, *, \diamond) \rightarrow\left(Y, \mu^{\prime}, v^{\prime}, *, \diamond\right)$ be a sequence of functions. Then for every $\varepsilon>0$, for each $x \in X$ and $t>0$, the following statements are equivalent:

(i) $I_{(\mu, v)}-f_{n} \rightarrow f$.

(ii) $\quad\left\{n \in \mathbb{N}: \mu^{\prime}\left(f_{n}(x)-f(x), t\right) \leq 1-\varepsilon\right\} \in I$ and $\left\{n \in \mathbb{N}: v^{\prime}\left(f_{n}(x)-f(x), t\right) \geq \varepsilon\right\} \in I$

(iii) $\left\{n \in \mathbb{N}: \mu^{\prime}\left(f_{n}(x)-f(x), t\right)>1-\varepsilon\right.$ and $\left.v^{\prime}\left(f_{n}(x)-f(x), t\right)<\varepsilon\right\} \in F(I)$

(iv) $\left\{n \in \mathbb{N}: \mu^{\prime}\left(f_{n}(x)-f(x), t\right)>1-\varepsilon\right\} \in F(I)$ and $\left\{n \in \mathbb{N}: v^{\prime}\left(f_{n}(x)-f(x), t\right)<\varepsilon\right\} \in F(I)$

(v) $\quad I-\lim \mu^{\prime}\left(f_{n}(x)-f(x), t\right)=1$ and $I-\lim v^{\prime}\left(f_{n}(x)-f(x), t\right)=0$

Proof. The proof is standard verification.

Theorem 1. Let $\left(f_{n}\right)$ and $\left(g_{n}\right)$ be two sequences of functions in an IFNS $(X, \mu, v, *, \diamond)$. If $I_{(\mu, v)}-f_{n}=f$ and $I_{(\mu, v)}-g_{n}=g$, then $I_{(\mu, v)}-\left(\alpha f_{n}+\beta g_{n}\right)=\alpha f+\beta g$ where $\alpha, \beta \in I F(\mathbb{R}$ or $\mathbb{C})$. 
Proof. The proof is clear for $\alpha=0$ and $\beta=0$. Now let $\alpha \neq 0$ and $\beta \neq 0$. Since $I_{(\mu, v)}-f_{n} \rightarrow f$ and $I_{(\mu, v)}-g_{n} \rightarrow g$, for each $x \in X$

$A_{1}=\left\{\begin{array}{r}n \in \mathbb{N}: \mu^{\prime}\left(f_{n}(x)-f(x), \frac{t}{2|\alpha|}\right) \leq 1-\varepsilon \\ \text { or } v^{\prime}\left(f_{n}(x)-f(x), \frac{t}{2|\alpha|}\right) \geq \varepsilon\end{array}\right\} \in I$,

and

$$
A_{2}=\left\{\begin{array}{r}
n \in \mathbb{N}: \mu^{\prime}\left(g_{n}(x)-g(x), \frac{t}{2|\beta|}\right) \leq 1-\varepsilon \\
\text { or } v^{\prime}\left(g_{n}(x)-g(x), \frac{t}{2|\beta|}\right) \geq \varepsilon
\end{array}\right\} \in I .
$$

Define the set $A=\left(A_{1} \cup A_{2}\right)$, so $A$ belongs to $I$. It follows that $A^{c}$ is a non-empty set in $F(I)$. We shall show that for each $x \in X$

$$
A^{c} \subset\left\{n \in \mathbb{N}: \mu^{\prime}\left(\left(\alpha f_{n}+\beta g_{n}\right)(x)-(\alpha f+\beta g)(x), t\right)\right.
$$
$>1-\varepsilon$ and $\left.v^{\prime}\left(\left(\alpha f_{n}+\beta g_{n}\right)(x)-(\alpha f+\beta g)(x), t\right)<\varepsilon\right\}$.

Let $m \in A^{c}$. In this case

$$
\begin{gathered}
\mu^{\prime}\left(f_{m}(x)-f(x), \frac{t}{2|\alpha|}\right)>1-\varepsilon, \\
v^{\prime}\left(f_{m}(x)-f(x), \frac{t}{2|\alpha|}\right)<\varepsilon, \\
\mu^{\prime}\left(g_{m}(x)-g(x), \frac{t}{2|\beta|}\right)>1-\varepsilon \\
\text { and } v^{\prime}\left(g_{m}(x)-g(x), \frac{t}{2|\beta|}\right)<\varepsilon .
\end{gathered}
$$

We have

$$
\begin{aligned}
& \mu^{\prime}\left(\left(\alpha f_{m}+\beta g_{m}\right)(x)-(\alpha f(x)+\beta g(x)), t\right) \\
& \quad \geq \mu^{\prime}\left(\alpha f_{m}(x)-\alpha f(x), \frac{t}{2}\right) * \mu^{\prime}\left(\beta g_{m}(x)-\beta g(x), \frac{t}{2}\right) \\
& \quad=\mu^{\prime}\left(f_{m}(x)-f(x), \frac{t}{2|\alpha|}\right) * \mu^{\prime}\left(g_{m}(x)-g(x), \frac{t}{2|\beta|}\right) \\
& \quad>(1-\varepsilon) *(1-\varepsilon) \\
& \quad=(1-\varepsilon)
\end{aligned}
$$

and

$$
\begin{aligned}
& v^{\prime}\left(\left(\alpha f_{m}+\beta g_{m}\right)(x)-(\alpha f(x)+\beta g(x)), t\right) \\
\leq & v^{\prime}\left(\alpha f_{m}(x)-\alpha f(x), \frac{t}{2}\right) * v^{\prime}\left(\beta g_{m}(x)-\beta g(x), \frac{t}{2}\right) \\
= & v^{\prime}\left(f_{m}(x)-f(x), \frac{t}{2|\alpha|}\right) * v^{\prime}\left(g_{m}(x)-g(x), \frac{t}{2|\beta|}\right) \\
< & \varepsilon \diamond \varepsilon \\
= & \varepsilon .
\end{aligned}
$$

This implies that

$$
A^{c} \subset\left\{n \in \mathbb{N}: \mu^{\prime}\left(\left(\alpha f_{n}+\beta g_{n}\right)(x)-(\alpha f+\beta g)(x), t\right)\right.
$$$$
\left.>1-\varepsilon \text { and } v^{\prime}\left(\left(\alpha f_{n}+\beta g_{n}\right)(x)-(\alpha f+\beta g)(x), t\right)<\varepsilon\right\} \text {. }
$$

Since $F(I)$ is filter, it follows that the later set belongs to $F(I)$. According to Lemma 1

$$
I_{(\mu, v)}-\left(\alpha f_{n}+\beta g_{n}\right) \rightarrow \alpha f+\beta g .
$$

Definition 9. Let $f_{n}:(X, \mu, v, *, \diamond) \rightarrow\left(Y, \mu^{\prime}, v^{\prime}, *, \diamond\right)$ be a sequence of functions. The sequence $\left(f_{n}\right)$ is a pointwise ideal Cauchy sequence in IFNS provided that for every $\varepsilon>$ 0 and $t>0$ there exists a number $N=N(\varepsilon, t, x)$ such that

$$
\left\{\begin{aligned}
n \in \mathbb{N}: \mu^{\prime}\left(f_{n}(x)-f_{N}(x), t\right) & \leq 1-\varepsilon \\
& \text { or } v^{\prime}\left(f_{n}(x)-f_{N}(x), t\right) \geq \varepsilon \text { for each } x \in X
\end{aligned}\right\} \in I .
$$

Theorem 2. Let $f_{n}:(X, \mu, v, *, \diamond) \rightarrow\left(Y, \mu^{\prime}, v^{\prime}, *, \diamond\right)$ be a sequence of functions. If $\left(f_{n}\right)$ is a pointwise I-convergent with respect to intuitionistic fuzzy norm $(\mu, v)$, then $\left(f_{n}\right)$ is a pointwise I-Cauchy sequence with respect to intuitionistic fuzzy norm $(\mu, v)$.

Proof. Suppose that $I_{(\mu, v)}-f_{n} \rightarrow f$ and let $\varepsilon>0, t>0$. For a given $\varepsilon>0$, choose $s>0$ such that $(1-\varepsilon) *(1-\varepsilon)>1-s$ and $\varepsilon \diamond \varepsilon<s$. Then for each $x \in X$,

$A_{x}(\varepsilon, t)=\left\{\begin{array}{r}n \in \mathbb{N}: \mu^{\prime}\left(f_{n}(x)-f(x), \frac{t}{2}\right) \leq 1-\varepsilon \\ \text { or } v^{\prime}\left(f_{n}(x)-f(x), \frac{t}{2}\right) \geq \varepsilon\end{array}\right\} \in I$

which implies that

$A_{x}^{c}(\varepsilon, t)=\left\{\begin{array}{r}n \in \mathbb{N}: \mu^{\prime}\left(f_{n}(x)-f(x), \frac{t}{2}\right)>1-\varepsilon \\ \text { and } v^{\prime}\left(f_{n}(x)-f(x), \frac{t}{2}\right)<\varepsilon\end{array}\right\} \in F(I)$.

Let us choose $N \in A_{x}^{c}(\varepsilon, t)$. Then

$\mu^{\prime}\left(f_{N}(x)-f(x), \frac{t}{2}\right)>1-\varepsilon$ and $v^{\prime}\left(f_{N}(x)-f(x), \frac{t}{2}\right)<\varepsilon$.

We want to show that there exists a number $N=N(x, \varepsilon, t)$ such that

$$
\left\{\begin{array}{c}
n \in \mathbb{N}: \mu^{\prime}\left(f_{n}(x)-f_{N}(x), t\right) \leq 1-s \\
\\
\text { or } v^{\prime}\left(f_{n}(x)-f_{N}(x), t\right) \geq s \text { for each } x \in X
\end{array}\right\} \in I .
$$

For this, define for each $x \in X$,

$$
B_{x}(\varepsilon, t)=\left\{\begin{array}{r}
n \in \mathbb{N}: \mu^{\prime}\left(f_{n}(x)-f_{N}(x), t\right) \leq 1-s \\
\text { or } v^{\prime}\left(f_{n}(x)-f_{N}(x), t\right) \geq s
\end{array}\right\} .
$$

We have to show that

$$
B_{x}(\varepsilon, t) \subset A_{x}(\varepsilon, t) .
$$

Suppose that

$$
B_{x}(\varepsilon, t) \nsubseteq A_{x}(\varepsilon, t) .
$$


In this case $B_{x}(\varepsilon, t)$ has at least one different element which $A_{x}(\varepsilon, t)$ doesn't has. Let $n \in B_{x}(\varepsilon, t) \backslash A_{x}(\varepsilon, t)$. Then we have

$$
\begin{array}{r}
\mu^{\prime}\left(f_{n}(x)-f_{N}(x), t\right) \leq 1-s \\
\text { and } \mu^{\prime}\left(f_{n}(x)-f(x), \frac{t}{2}\right)>1-\varepsilon,
\end{array}
$$

in particularly $\mu^{\prime}\left(f_{N}(x)-f(x), \frac{t}{2}\right)>1-\varepsilon$. In this case

$$
\begin{aligned}
1-s & \geq \mu^{\prime}\left(f_{n}(x)-f_{N}(x), t\right) \\
& \geq \mu^{\prime}\left(f_{n}(x)-f(x), \frac{t}{2}\right) * \mu^{\prime}\left(f_{N}(x)-f(x), \frac{t}{2}\right) \\
& \geq(1-\varepsilon) *(1-\varepsilon)>1-s,
\end{aligned}
$$

which is not possible. On the other hand

$v^{\prime}\left(f_{n}(x)-f_{N}(x), t\right) \geq s$ and $v^{\prime}\left(f_{n}(x)-f(x), t / 2\right)<\varepsilon$, in particularly $v^{\prime}\left(f_{N}(x)-f(x), t / 2\right)<\varepsilon$. In this case

$$
\begin{gathered}
s \leq v^{\prime}\left(f_{n}(x)-f_{N}(x), t\right) \\
\leq v^{\prime}\left(f_{n}(x)-f(x), \frac{t}{2}\right) \diamond v^{\prime}\left(f_{N}(x)-f(x), \frac{t}{2}\right) \\
<\varepsilon \diamond \varepsilon<s
\end{gathered}
$$

which is not possible. Hence $B_{x}(\varepsilon, t) \subset A_{x}(\varepsilon, t)$. Therefore, since $A_{x}(\varepsilon, t) \in I, B_{x}(\varepsilon, t) \in I,\left(f_{n}\right)$ is a pointwise $I-$ Cauchy sequence with respect to intuitionistic fuzzy norm $(\mu, v)$ on $X$.

In studying on sequences of functions, uniform convergence is an other important concept. Now we introduce uniformly $I$-convergence of sequences of functions in an IFNS. Let us start with the following definition.

Definition 10. Let $(X, \mu, v, *, \diamond)$ and $\left(Y, \mu^{\prime}, v^{\prime}, *, \diamond\right)$ be two IFNS and $f_{n}:(X, \mu, v, *, \diamond) \rightarrow\left(Y, \mu^{\prime}, v^{\prime}, *, \diamond\right)$ be a sequence of functions. If for every $x \in X$ and $\forall \varepsilon>0, t>0$,

$$
\begin{array}{r}
\left\{n \in \mathbb{N}: \mu^{\prime}\left(f_{n}(x)-f(x), t\right) \leq 1-\varepsilon\right. \\
\left.\quad \text { or } v^{\prime}\left(f_{n}(x)-f(x), t\right) \geq \varepsilon\right\} \in I,
\end{array}
$$

then we say that the sequence $\left(f_{n}\right)$ is uniformly I-convergent with respect to intuitionistic fuzzy norm $(\mu, v)$ and we denote $I_{(\mu, v)}-f_{n} \rightrightarrows f$.

Corollary 1. Let $I=\{A \subset \mathbb{N}: A$ is a finite set $\}$. Then $I$ is an admissible ideal in $\mathbb{N}$. Hence, in the Definition 8 and the Definition 10, I-convergence coincides usual convergence of sequences of functions with respect to intuitionistic fuzzy norm.

Corollary 2. Let $I=\{A \subset \mathbb{N}: \delta(A)=0\}$. Then $I$ is an admissible ideal in $\mathbb{N}$. Hence, in the Definition 8 and the Definition 10, I-convergence coincides with statistical convergence of sequences of function with respect to intuitionistic fuzzy norm.
Remark. Let $f_{n}:(X, \mu, v, *, \diamond) \rightarrow\left(Y, \mu^{\prime}, v^{\prime}, *, \diamond\right)$ be a sequence of functions. If $\left(f_{n}\right)$ is uniformly intuitionistic fuzzy convergent on $X$ to a function $f$ with respect to $(\mu, v)$, then $I_{(\mu, v)}-f_{n} \rightrightarrows f$. But the converse of this is not true.

Remark. If $I_{(\mu, v)}-f_{n} \rightrightarrows f$, then $I_{(\mu, v)}-f_{n} \rightarrow f$.

Example 2. Let $I$ be as in Example 1 and consider an intuitionistic fuzzy norm on $\mathbb{R}$ as in Example 1. Let $f_{n}:[0,1) \rightarrow \mathbb{R}$ be sequence of functions whose terms are given by

$$
f_{n}(x)=\left\{\begin{array}{cc}
x^{n}+2 ; & \text { if } n=2 k+1(n \in \mathbb{N}) \\
1 ; & \text { otherwise }
\end{array}\right.
$$

Then, for every $0<\varepsilon<1$ and for every $t>0$, we have

$$
\begin{aligned}
K(\varepsilon, t)= & \left\{n \in \mathbb{N}: \mu\left(f_{n}(x)-f(x), t\right) \leq 1-\varepsilon\right. \\
& \text { or } \left.v\left(f_{n}(x)-f(x), t\right) \geq \varepsilon\right\} \\
K(\varepsilon, t)= & \left\{n \in \mathbb{N}: \frac{t}{t+\left|f_{n}(x)-1\right|} \leq 1-\varepsilon\right. \\
& \text { or } \left.\frac{\left|f_{n}(x)-1\right|}{t+\left|f_{n}(x)-1\right|} \geq \varepsilon\right\} \\
= & \left\{n \in \mathbb{N}:\left|f_{n}(x)-1\right| \geq \frac{\varepsilon t}{1-\varepsilon}\right\} \\
= & \left\{n \in \mathbb{N}: f_{n}(x)=x^{n}+2\right\} \\
= & \{n \in \mathbb{N}: n=2 k+1\} .
\end{aligned}
$$

Since $K(\varepsilon, t) \in I$, then the sequence $\left(f_{n}\right)$ is uniformly $I$-convergent to 1 with respect to $(\mu, v)$ for all $x \in X_{0}$.

Remark. In the example above, the sequence $\left(f_{n}\right)$ is not uniform convergent both usual sense and statistical sense with respect to $(\mu, v)$ but it is uniform $I$-convergent with respect to $(\mu, v)$.

Lemma 2. Let $f_{n}:(X, \mu, v, *, \diamond) \rightarrow\left(Y, \mu^{\prime}, v^{\prime}, *, \diamond\right)$ be a sequence of functions. Then for every $\varepsilon>0$, for every $x \in$ $X$ and $t>0$, the following statements are equivalent:

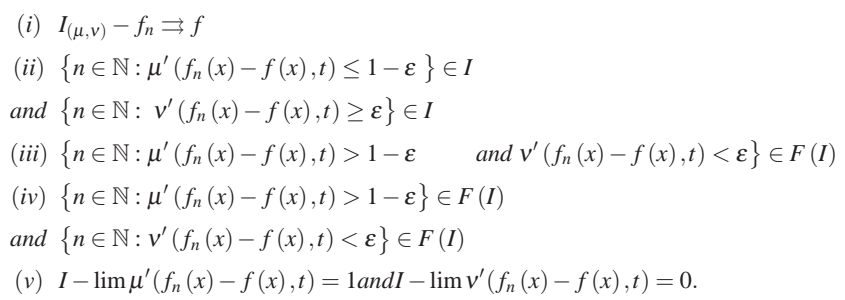

Definition 11. Let $f_{n}:(X, \mu, v, *, \diamond) \rightarrow\left(Y, \mu^{\prime}, v^{\prime}, *, \diamond\right)$ be a sequence of functions. The sequence $\left(f_{n}\right)$ is a uniform ideal Cauchy sequence in intuitionistic fuzzy normed space provided that for every $\varepsilon>0$ and $t>0$ there exists a number $N=N(\varepsilon, t)$ such that

$$
\left\{\begin{array}{c}
n \in \mathbb{N}: \mu^{\prime}\left(f_{n}(x)-f_{N}(x), t\right) \leq 1-\varepsilon \text { or } \\
v^{\prime}\left(f_{n}(x)-f_{N}(x), t\right) \geq \varepsilon \text { for every } x \in X
\end{array}\right\} \in I .
$$


Theorem 3. Let $f_{n}:(X, \mu, v, *, \diamond) \rightarrow\left(Y, \mu^{\prime}, v^{\prime}, *, \diamond\right)$ be a sequence of functions. If $\left(f_{n}\right)$ is a uniformly Iconvergent sequence with respect to intuitionistic fuzzy norm $(\mu, v)$, then $\left(f_{n}\right)$ is uniformly I- convergent Cauchy sequence with respect to intuitionistic fuzzy norm $(\mu, v)$.

Proof. The proof is similar to the Theorem 2. We omit it.

Theorem 4. Let $(X, \mu, v, *, \diamond)$ and $\left(Y, \mu^{\prime}, v^{\prime}, *, \diamond\right)$ be two IFNS and $f_{n}:(X, \mu, v, *, \diamond) \rightarrow\left(Y, \mu^{\prime}, v^{\prime}, *, \diamond\right)$ be an intuitionistic fuzzy continuous mapping on $X$. If $I_{(\mu, v)}-f_{n} \rightarrow f$, then $f: X \rightarrow Y$ is an intuitionistic fuzzy continuous mapping on $X$.

Proof. Let $x_{0} \in X$ be an arbitrary point. By the intuitionistic continuity of $f_{n}$ 's, there exist $\delta>0$ such that $\mu\left(x-x_{0}, t\right)>1-\delta$ and $v\left(x-x_{0}, t\right)<\delta \Rightarrow$

$\mu^{\prime}\left(f_{n}\left(x_{0}\right)-f_{n}(x), t\right)>1-\varepsilon$ and $v^{\prime}\left(f_{n}\left(x_{0}\right)-f_{n}(x), t\right)<\varepsilon$

for every $n \in \mathbb{N}$ and $t>0$. Let $x \in B\left(x_{0}, \delta, t\right)$ (open ball with center $x_{0}$ and radius $\delta$ in intuitionistic fuzzy normed space $(X, \mu, v, *, \diamond))$ be fixed. Since $I_{(\mu, v)}-f_{n} \rightarrow f$ on $X$, the sets

$A=\left\{\begin{array}{c}n \in \mathbb{N}: \mu^{\prime}\left(f_{n}(x)-f(x), \frac{t}{3}\right) \leq 1-\varepsilon \\ \quad \text { or } v^{\prime}\left(f_{n}(x)-f(x), \frac{t}{3}\right) \geq \varepsilon \text { for each } x \in X\end{array}\right\} \in I$

and

$B=\left\{\begin{array}{c}n \in \mathbb{N}: \mu^{\prime}\left(f_{n}\left(x_{0}\right)-f\left(x_{0}\right), \frac{t}{3}\right) \leq 1-\varepsilon \\ \quad \text { or } v^{\prime}\left(f_{n}\left(x_{0}\right)-f\left(x_{0}\right), \frac{t}{3}\right) \geq \varepsilon \text { for each } x \in X\end{array}\right\} \in I$

is in $I$ so $A \cup B \in I$ and $A \cup B$ is different from $\mathbb{N}$ since $I$ is non-trivial. Thus, there exists an $n_{0} \in \mathbb{N}$ such that

$$
\begin{array}{r}
\mu^{\prime}\left(f_{n_{0}}(x)-f(x), \frac{t}{3}\right)>1-\varepsilon, \\
v^{\prime}\left(f_{n_{0}}(x)-f(x), \frac{t}{3}\right)<\varepsilon
\end{array}
$$

and

$$
\begin{array}{r}
\mu^{\prime}\left(f_{n_{0}}\left(x_{0}\right)-f\left(x_{0}\right), \frac{t}{3}\right)>1-\varepsilon, \\
v^{\prime}\left(f_{n_{0}}\left(x_{0}\right)-f\left(x_{0}\right), \frac{t}{3}\right)<\varepsilon
\end{array}
$$

Now, we will show that $f$ is intuitionistic fuzzy continuous at $x_{0}$. We have

$$
\begin{aligned}
\mu^{\prime}\left(f(x)-f\left(x_{0}\right), t\right)= & \mu^{\prime}\left(f(x)-f_{n_{0}}(x)+f_{n_{0}}(x)\right. \\
& \left.-f_{n_{0}}\left(x_{0}\right)+f_{n_{0}}\left(x_{0}\right)-f\left(x_{0}\right), t\right) \\
\geq & \mu^{\prime}\left(f(x)-f_{n_{0}}(x), \frac{t}{3}\right) \\
& * \mu^{\prime}\left(f_{n_{0}}(x)-f_{n_{0}}\left(x_{0}\right), \frac{t}{3}\right) \\
& * \mu^{\prime}\left(f_{n_{0}}\left(x_{0}\right)-f\left(x_{0}\right), \frac{t}{3}\right) \\
> & (1-\varepsilon) *(1-\varepsilon) *(1-\varepsilon) \\
= & 1-\varepsilon
\end{aligned}
$$

and

$$
\begin{aligned}
v^{\prime}\left(f(x)-f\left(x_{0}\right), t\right)= & v^{\prime}\left(f(x)-f_{n_{0}}(x)+f_{n_{0}}(x)\right. \\
& \left.-f_{n_{0}}\left(x_{0}\right)+f_{n_{0}}\left(x_{0}\right)-f\left(x_{0}\right), t\right) \\
\leq & v^{\prime}\left(f(x)-f_{n_{0}}(x), \frac{t}{3}\right) \\
& \diamond v^{\prime}\left(f_{n_{0}}(x)-f_{n_{0}}\left(x_{0}\right), \frac{t}{3}\right) \\
& \diamond v^{\prime}\left(f_{n_{0}}\left(x_{0}\right)-f\left(x_{0}\right), \frac{t}{3}\right) \\
< & \varepsilon \diamond \varepsilon \diamond \varepsilon \\
= & \varepsilon .
\end{aligned}
$$

Hence the proof is completed.

Acknowledgements : This work is supported by The Scientific and Technological Research Council of Turkey (TUBITAK) under the project number 110T699.

\section{References}

[1] K. Atanassov, Intuitionistic fuzzy sets, Fuzzy Sets and Systems, 20, 87-96 (1986).

[2] M. Balcerzak, K. Dems, A. Komisarski, Statistical convergence and ideal convergence for sequences of function, J. Math. Anal. Appl., 328, 715-729 (2007).

[3] L.C. Barros, R.C. Bassanezi, P.A. Tonelli, Fuzzy modelling in population dynamics, Ecol. Model., 128, 27-33 (2000).

[4] B. Dinda, T.K. Samanta, Intuitionistic Fuzzy Continuty and Uniform Convergence, Int. J. Open Problems Compt., Math., 3, (2010).

[5] H. Fast, Surla convergence statistique, Colloq. Math., 2, 241-244 (1951).

[6] A.L. Fradkov, R.J. Evans, Control of chaos: Methods and applications in engineering, Chaos Solitons Fractals, 29, 33 56 (2005).

[7] A. George, P. Veeramani, On some results in fuzzy metric spaces, Fuzzy Sets Systems, 64, 395-399 (1994).

[8] R. Giles, A computer program for fuzzy reasoning, Fuzzy Sets and System, 4, 221-234 (1980).

[9] L. Hong, J.Q. Sun, Bifurcations of fuzzy nonlinear dynamical systems, Commun. Nonlinear Sci. Numer. Simul., 1, 1-12 (2006).

[10] V. Karakaya, N. Şimşek, M. Ertürk and F. Gürsoy, Statistical convergence of sequences of functions in intuitionistic fuzzy normed spaces, Abstract and Applied Analysis, 2012 (2012).

[11] V. Karakaya, N. Şimşek, M. Ertürk and F. Gürsoy, $\lambda$-statistical convergence of sequences of functions in intuitionistic fuzzy normed spaces, Journal of Function Spaces and Applications, (2012),

[12] V. Karakaya, N. Şimşek, M. Ertürk and F. Gürsoy, Lacunary statistical convergence of sequences of functions in intuitionistic fuzzy normed space,Journal of Intelligent and Fuzzy Systems, doi "10.3233/IFS-130815" (2013).

[13] S. Karakuş, K. Demirci, O. Duman, Statistical convergence on intuitionistic fuzzy normed spaces, Chaos Solitons and Fractals, 35, 763-69 (2008). 
[14] A. Komisaski, Pointwise $I$-convergence and $I^{*}$.convergence in measure of sequences of functions, J. Math. Anal. Appl., 340, 770-779 (2008).

[15] P.Kostyrko, T. Šalăt and W. Wilczyňski, $I$-Convergence, Real Analysis Exchange, 26, 669-686 (2000/2001).

[16] V. Kumar and K. Kumar, On Ideal Convergence of Sequences in Intuitionistic Fuzzy Normed Spaces, Selçuk Journal of Applied Mathematics, 10, 27-36 (2009).

[17] J. Madore, Fuzzy physics, Ann. Phys., 219, 187-198 (1992).

[18] M. Mursaleen, V. Karakaya and S. A. Mohiuddine, Schauder Basis, Separability, and Approximation Property in Intuitionistic Fuzzy Normed Space, Abstract and Applied Analysis, 2010, Article ID 131868, 14 pages.

[19] M. Mursaleen, S. A. Mohiuddine, Osama H. H. Edely, On the ideal convergence of double sequences in intuitionistic fuzzy normed spaces, Comput. Math. Appl., 59, 603-611 (2010).

[20] M. Mursaleen and A. Alotaibi, On I-convergence in random 2-normed spaces, Math. Slovaca, 61, 933-940 (2011).

[21] M. Mursaleen and S.A. Mohiuddine, On ideal convergence in probabilistic normed spaces, Math. Slovaca, 62, 49-62 (2012).

[22] M. Mursaleen and S.A. Mohiuddine, Statistical convergence of double sequences in intuitionistic fuzzy normed spaces, Chaos Solitons and Fractals, 41, 2414-2421 (2009).

[23] M. Mursaleen and Osama H.H.Edely, Statistical convergence of double sequences, J. Math. Anal. Appl., 288, 223-231 (2003).

[24] P. Das, Fuzzy topology on fuzzy sets: product fuzzy topology and fuzzy topological groups, Fuzzy Sets and Systems, 100, 367-372 (1998).

[25] J.H Park., Intuitionistic fuzzy metric spaces, Chaos Solitos and Fractals, 22, 1039-1046 (2004).

[26] R. Saadati, J.H. Park, On the intuitionistic fuzzy topological spaces, Chaos Solitons and Fractals, 27, 331-44 (2006).

[27] T. K. Samanta and I. H. Jebril, Finite dimensional Intuitionistic fuzzy normed spaces, Int. J. Open problems Compt. Math., 2, 574-591 (2009).

[28] B. Schweizer, A. Skalar, Statistical metric spaces, Pasific J. Math., 10, 13-34 (1960).

[29] L.A. Zadeh, Fuzzy Sets, Inform. Control, 8, 338-353 (1965).

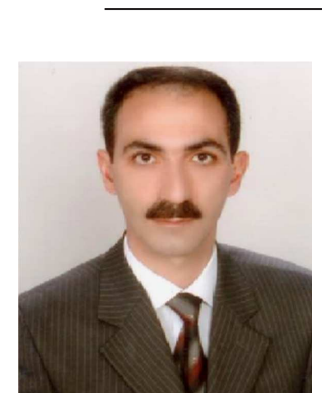

Vatan Karakaya was born in Malatya-Turkey, in 1971. He received $\mathrm{Ph}$. D. degree in Analysis and Functions Spaces. His research interests include summability, sequence and series, the geometry of Banach spaces, the operator theory and spectral properties, intuitionistic fuzzy spaces. At the present, he is Professor of Mathematical Engineering at Yildiz Technical University in İstanbul-Turkey.

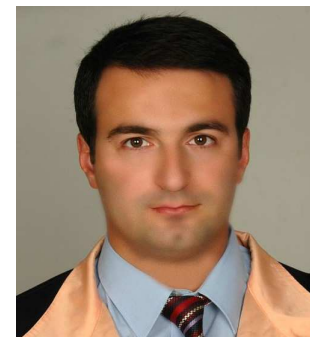

Necip Şimşek is received the $\mathrm{Ph}$. D. degree in Mathematical Analysis. His research interests include geometry of Banach sequence spaces. At the present he is researcher at İstanbul Commerce University, İstanbul-Turkey.

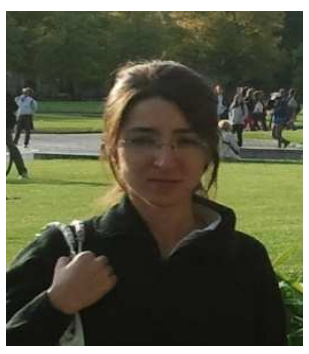

Müzeyyen ERTÜRK Graduated from Harran University, Turkey in 2009 with MSc. in Functional Analysis. She is currently taking a $\mathrm{PhD}$ under the supervision of Prof. Dr. Vatan Karakaya and co supervisor Prof. Dr. Mohammed Mursaleen. She has an interest in the field of inuitionistic fuzzy normed space and fixed point theory. She is now a research assistant in the Department of Mathematics in Yildiz Technical University, İstanbul-Turkey.

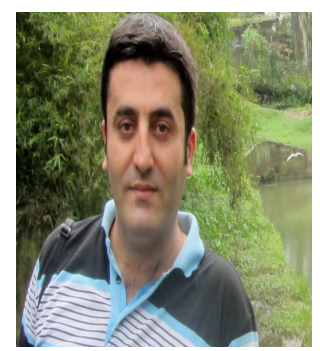

Faik GÜRSOY serves as research assistant for the Department of Mathematics at the Yildiz Technical University, İstanbul-Turkey. $\mathrm{He}$ is currently pursuing a Ph.D. degree in mathematics at the Yildiz Technical University since spring of 2010 under the co-supervision of Professor Vatan Karakaya and Professor Billy E. Rhoades. He received his B.S. in Mathematics from İnönü University in June of 2004 and then received an M.S. in Mathematics from Harran University in August of 2008 under the supervision of Associate Professor Necip Simsek. Faik's current research interests include Fixed Point Theory and Intuitionistic Fuzzy Normed Spaces. 